ERRAT U M

\title{
Healthcare resource utilization and cost in dementia: are there differences between patients screened positive for dementia with and those without a formal diagnosis of dementia in primary care in Germany? - ERRATUM
}

Bernhard Michalowsky, Tilly Eichler, Jochen René Thyrian, Johannes Hertel, Diana Wucherer, Wolfgang Hoffmann and Steffen Flessa

http://dx.doi.org/10.1017/S1041610215001453, Published online by Cambridge University Press 22 September 2015.

In the above mentioned article by Michalowsky et al., Johannes Hertel was mistakenly omitted from the authorship list. This error has been corrected in the print, PDF and HTML versions of the original article.

\section{Reference}

Michalowsky, B. et al. Healthcare resource utilization and cost in dementia: are there differences between patients screened positive for dementia with and those without a formal diagnosis of dementia in primary care in Germany. International Psychogeriatrics, published online 22 September 2015, http://dx.doi.org/10.1017/S1041610215001453. 